\title{
FutureJournal
}

\section{Critérios para Identificação da Saliência de Stakeholders através da Análise de Conteúdo}

\author{
João Maurício Gama Boaventura \\ Universidade de São Paulo, USP/FEA, Brasil \\ jboaventura@usp.br \\ Luis Guilherme Paulon Fontes \\ Universidade de São Paulo, USP/FEA, Brasil \\ Igpfontes@gmail.com

\section{Greici Sarturi} \\ Universidade Federal de Santa Maria (UFSM), Brasil \\ greici@usp.br

\section{Eduardo Armando} \\ Faculdade FIA de Administração e Negócios (FFIA), Brasil \\ earmando@usp.br
}

\section{RESUMO}

É notória a integração da Teoria dos Stakeholders à área de Estratégia Empresarial, evidência explícita é o título da obra seminal desta teoria, a de Freeman (1984): Strategic Management - a stakeholder approach. Desde então, o campo de Stakeholder Strategy evoluiu consideravelmente em termos conceituais e instrumentais. Vários modelos foram desenvolvidos, entre eles o modelo de saliência de stakeholders proposto por Mitchell et al. (1997). Esta pesquisa contribui para o campo de Stakeholder Strategy ao ter como objetivo desenvolver uma metodologia de análise de conteúdo para a análise da saliência de stakeholders. O modelo de Saliência baseia-se na percepção dos gestores quanto a posse dos atributos Poder, Legitimidade e Urgência pelos stakeholders. A combinação entre os três atributos gera 7 classes de stakeholders. O método proposto neste estudo é composto de seis etapas: 1) definição da fonte de dados, 2) definição das seções do documento a serem analisadas, 3) definição das palavras chave, 4) critérios de associação entre stakeholder e atributo, 5) critérios para identificação da presença do atributo, 6) processamento da análise. Os resultados indicaram que a metodologia é operacionalmente aplicável, uma vez que foi possível identificar, na amostra testada, as classes de stakeholders propostas por Mitchell et al. (1997). A pesquisa apresenta uma contribuição metodológica para a análise do modelo de saliência o que pode auxiliar os pesquisadores no acesso a uma maior 


\section{FutureJournal}

quantidade de informações sobre a percepção dos gestores em relação aos stakeholders.

PALAVRAS-ChAVE: Análise de conteúdo. Saliência. Gestão de stakeholders.

\section{Criteria for Identication of Stakeholders Salience through Content Analysis}

\section{ABSTRACT}

It is notorious the integration of the Stakeholders Theory to the area of Corporate Strategy, an explicit evidence of this is the title of the seminal work of this theory: Strategic Management - the stakeholder approach by Freeman (1984). Since then, the Stakeholder Strategy field has evolved considerably in conceptual and instrumental terms. Several models have been developed, among them the Salience Stakeholder Model proposed by Mitchell et al. (1997). This research contributes to the Stakeholder Strategy field aiming atdeveloping $\mathrm{p}$ a method of content analysis to examine stakeholder saliency. The salience model was proposed by Mitchell et al. (1997) and is based on the managerial perception on stakeholders having the following attributes: Power, Legitimacy and Urgency. The combination of these three attributes generates seven classes of stakeholders. The methodology used in this research is composed of six steps: 1) data source definition, 2) definition of the parts of the document text that should be examined, 4) criteria for associating stakeholders and attributes, 5) criteria for identifying attribute presence, 6) running the analysis. Results indicate that methodology is operationally feasible because it was possible to observe in the tested sample the stakeholder classes proposed by Mitchell et al. (1997). The main contribution of this investigation is methodological, focusing on the salience model analysis. This contribution may support researchers in accessing larger databases on managerial perception regarding stakeholders.

KEY-WORDS: Content analysis. Salience. Stakeholder management. 


\section{INTRODUÇÃO}

A teoria dos stakeholders passou definitivamente a fazer parte da estratégia empresarial a partir de 1984 com a obra seminal de Freeman (1984), Strategic management: a stakeholder approach. Com o desenvolvimento desta teoria, surgiu o campo de stakeholder strategy, o qual figura no Strategic Management Society como uma divisão de estudos. Desde então, este campo de estudos evoluiu consideravelmente em termos conceituais, e vários modelos de análise foram desenvolvidos. Diversos autores contribuíram para o enriquecimento da literatura $\mathrm{e}$ da operacionalização do conceito de stakeholders. Além da evolução acadêmica, diversas empresas já adotam os conceitos da teoria do stakeholder no seu dia a dia, como pode ser percebido pela presença do mapa de stakeholders em alguns relatórios corporativos, como o relatório de responsabilidade social empresarial (Weber \& Marley, 2012).

Uma dessas evoluções na teoria dos stakeholders foi 0 desenvolvimento do modelo de saliência de stakeholders proposto por Mitchell, Agle e Wood (1997), que se baseia na percepção dos gestores quanto à posse dos atributos poder, legitimidade e urgência pelos stakeholders. A proposição de Mitchell et al. (1997) contribuiu para a discussão sobre a identificação e a priorização de stakeholders e foi bem aceito pelos acadêmicos, dada a quantidade de artigos que versam sobre esse tema (Parent \& Deephouse, 2007).

Neville, Bell e Whitwell (2011) apontam o modelo de saliência como uma das contribuições mais significativas para o desenvolvimento das pesquisas de stakeholders. Contudo, a saliência é um construto de difícil mensuração e não há um método padrão para a investigação empírica do modelo. Os estudos que o empregaram empiricamente são pouco detalhados, o que indica a existência de uma lacuna metodológica referente à mensuração do modelo.

Um dos possíveis caminhos para a realização de pesquisas empíricas sobre saliência é a análise de conteúdo de relatórios corporativos. Esses relatórios, sejam eles prospectos de distribuição de ações, relatórios de sustentabilidade social empresarial, sejam relatórios contábeis, são valiosos 
em informações sobre a empresa e podem conter informações que demonstrem a percepção dos gestores sobre os stakeholders das organizações.

As atuais técnicas de levantamento e análise de dados podem fornecer instrumentos robustos para pesquisas empíricas que tenham como objetivo a identificação da saliência dos stakeholders. Alguns exemplos são os estudos de Weber e Marley (2012) e Bandiera, Boaventura, Mascena e Fishmann (2013), que utilizaram técnicas de análise de conteúdo para a identificação da saliência. Bandiera et al. (2013), mais especificamente, propõem uma metodologia para análise da saliência em prospectos definitivos através do cálculo da distância entre a palavra que representa o atributo (poder, legitimidade ou urgência) e a palavra que representa o stakeholder.

Embora os autores tenham contribuído metodologicamente para o avanço do modelo ao empregar um método quantitativo de análise de conteúdo para estudo da saliência, observam-se alguns aspectos que demandam melhorias, entre eles, o critério de definição das palavras utilizadas para caracterização dos atributos, assim como os critérios de busca utilizados.

Em função desta lacuna metodológica do modelo de saliência e das limitações do método proposto por Bandiera et al. (2013), nesta pesquisa o objetivo é desenvolver uma metodologia de análise de conteúdo para o modelo de saliência.

\section{REVISÃO TEÓRICA}

\subsection{TEORIA DOS STAKEHOLDERS}

A teoria dos stakeholders foi apresentada e utilizada de diversas maneiras e sob diferentes metodologias. Donaldson e Preston (1995), ao analisarem a literatura acadêmica sobre a teoria, propuseram que seu desenvolvimento pode ser classificado em três dimensões: descritiva/empírica, instrumental e normativa.

A dimensão descritiva é aquela em que a teoria é utilizada para descrever e, às vezes, explicar características e comportamentos específicos 
da corporação. A dimensão instrumental é aquela em que a teoria, em conjunto com dados empíricos, é utilizada para identificar conexões, ou ausência destas, entre a gestão de stakeholders e a realização de objetivos corporativos tradicionais (e.g., lucro, crescimento). Na dimensão normativa, a teoria é utilizada para interpretar a função da empresa, incluindo a identificação de diretrizes morais ou filosóficas para operação e gestão das empresas (Donaldson \& Preston, 1995).

Clarkson (1995) e Freeman (1984) afirmam a necessidade de entender quem são e qual a importância dos stakeholders como primeiro passo de uma análise de stakeholder. Embora se encontrem na literatura algumas tentativas de identificar e classificar os stakeholders, Phillips e Reichart (2000) argumentam que a teoria é, muitas vezes, incapaz de distinguir entre os indivíduos e grupos que são ou não são stakeholders. Nesse contexto, Mitchell et al. (1997) propuseram o modelo de saliência, cujo propósito é identificar quem são os stakeholders da empresa e para quem os gestores devem destinar sua atenção.

Na próxima seção será apresentado o modelo de saliência proposto por Mitchell et al. (1997).

\subsection{MODELO DE SALIÊNCIA}

O modelo de saliência apresenta a importância percebida pelo gestor em relação a cada stakeholder em função da posse de um, dois ou todos os seguintes atributos: (1) o poder do stakeholder de influenciar a empresa; (2) a legitimidade do relacionamento entre o stakeholder e a empresa; e (3) a urgência da reivindicação do stakeholder para com a empresa.

Mitchell et al. (1997) afirmam que o stakeholder não possui de forma estática um atributo, ou seja, os atributos têm um status variável e o stakeholder pode não ter consciência de seus atributos ou não os estar exercendo.

Assim, pode-se determinar o grau de saliência dos stakeholders a partir da combinação dos três atributos de saliência - poder, legitimidade e urgência - como percebidos pelos gestores (Mitchell et al., 1997). Na Figura 1 , ilustram-se as possíveis combinações, sendo a saliência diretamente 
proporcional ao número de atributos que o gestor percebe que o stakeholder possui.

A combinação entre os três atributos gera sete classes de stakeholders (Mitchell et al., 1997). Os stakeholders que possuem apenas um atributo são considerados de baixa saliência, ou "latentes". Eles podem ser classificados em "adormecidos", quando possuem apenas o atributo poder; "arbitrários" se possuem somente legitimidade; ou "reivindicadores" se seu único atributo é a urgência.

Já os stakeholders a quem são atribuídos dois atributos podem ser considerados de saliência moderada ou "expectantes". Estes também podem ser divididos em três grupos: o "dominante", que tem sua influência na empresa assegurada pelo poder e pela legitimidade; o "perigoso", pois possui poder e urgência, mas sem legitimidade; e o "dependente", que tem alegações de legitimidade e urgência, mas depende do poder de outros stakeholders para ter suas reivindicações consideradas.

Os stakeholders percebidos como detentores de todos os atributos são os altamente salientes, denominados stakeholders "definitivos".

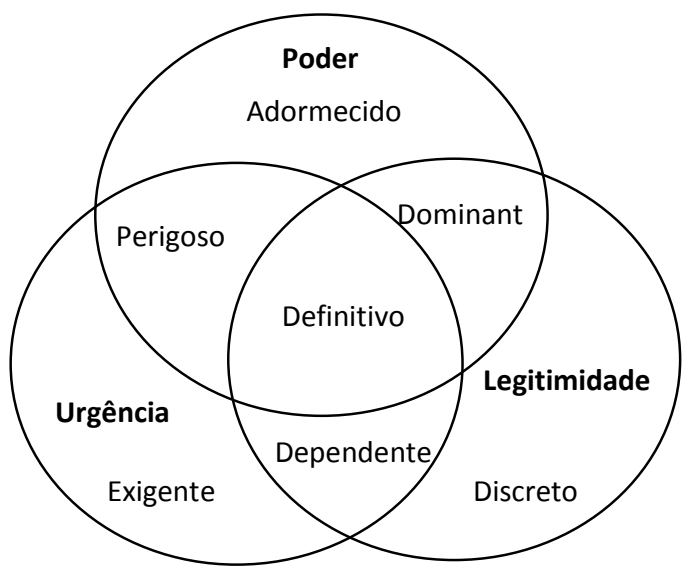

\section{Figura 1: Tipologia dos stakeholders}

Fonte: Mitchell et al. (1997, p. 87)

\subsubsection{Atributo Poder}

O primeiro atributo em que a saliência do stakeholder será analisada é o de poder. As atuais definições de poder derivam, ao menos em parte, da ideia propagada pelo sociólogo Weber (1947) de que poder é a probabilidade 
de um agente em um relacionamento social estar em uma posição de impor sua vontade apesar de resistência. Pfeffer (1981, citado por Mitchell et al., 1997, p. 865) utiliza Dahl (1957, p. 3) para definir poder como um relacionamento entre agentes sociais em que um agente social $A$ consegue que outro agente social B faça algo que B não faria. Mitchell et al. (1997) concordam com essas definições de poder para este atributo do stakeholder e, além disso, utilizam uma classificação de poder formulada por Etzioni (1964).

Etzioni (1964) sugere uma lógica para categorização do poder no ambiente organizacional baseada no tipo de recurso utilizado para seu exercício. Segundo esse autor, são três as categorias de poder: coercitivo, utilitário e simbólico. O poder coercitivo é exercido por meio da aplicação de recursos físicos; o poder utilitário, por meio de recursos materiais como premiações em dinheiro; e o poder simbólico, por meio de recursos simbólicos que podem ser normativos (como prestígio e estima) e sociais (como aprovação social e tradição familiar).

\subsubsection{Atributo Legitimidade}

O segundo atributo que um stakeholder pode possuir é legitimidade. Mitchell et al. (1997) utilizam a definição de Suchman (1995), segundo a qual legitimidade é uma percepção generalizada ou uma presunção de que as ações de uma entidade são desejáveis, adequadas, ou apropriadas dentro de um sistema social de normas, valores, crenças e definições. Apesar de utilizarem a definição de Suchman (1995) para o atributo legitimidade, os autores classificam legitimidade conforme o proposto por Wood (1991).

Wood (1991) classifica legitimidade nos níveis individual, organizacional e institucional. O nível individual é aquele das responsabilidades morais dos gestores, pois é esperado deles, como agentes morais, que sigam critérios desejáveis para a organização. O nível organizacional é o nível da empresa, no qual estão as regras e os códigos de conduta que a empresa possui. Por último vem o nível institucional, que é o da relação entre empresa e sociedade, no qual estão as leis que regem as 
atividades das empresas e as definições do que é esperado que a empresa entregue para a sociedade.

\subsubsection{Atributo Urgência}

O último atributo para classificação de saliência é o de urgência. Esse atributo torna-se necessário, pois poder e legitimidade, segundo Mitchell et al. (1997), não respondem por si sós a toda a dinâmica das interações entre stakeholders e gerentes.

Mitchell et al. (1997) entendem a urgência como o grau em que a reivindicação do stakeholder demanda atenção imediata e que urgência só existe em duas condições: (1) sensibilidade a prazo e (2) criticidade, isto é, quando o relacionamento ou reivindicação é crítico para o stakeholder.

\subsection{APLICAÇÃO DO MODELO DE SALIÊNCIA EM ESTUDOS EMPÍRICOS}

Agle, Mitchell e Sonnenfeld (1999) realizaram um estudo empírico sobre a saliência dos stakeholders em que tentavam estabelecer uma relação entre saliência de stakeholders e variáveis como valores dos gestores e performance corporativa. Com base em entrevistas, eles examinaram a percepção dos CEOs sobre os atributos de poder, legitimidade, urgência e como isso se refletia na saliência dos stakeholders. Os autores esclarecem que a percepção dos gestores é um fator crítico para a análise de saliência (Agle et al., 1999). Os resultados do estudo confirmam a validade do modelo de saliência de stakeholders proposto por Mitchell et al. (1997), mas não conseguem afirmar com segurança a existência de uma relação entre saliência percebida e performance. Como considerações finais, os autores recomendam mais pesquisas sobre o tema.

No contexto brasileiro, Xavier, Vieira e Costa (2011) identificaram o grau de saliência dos stakeholders de uma instituição bancária, empregando entrevistas com os gestores. O resultado da pesquisa apontou que os convênios, órgãos reguladores, parceiros financeiros e o banqueiro/acionistas são stakeholders definitivos (possuem os três atributos). Os clientes, funcionários e correspondentes exclusivos são stakeholders dominantes (com 
os atributos de legitimidade e poder). E os correspondentes preferenciais foram classificados como perigosos (atributos de poder e urgência).

Weber e Marley (2012) identificaram a saliência dos stakeholders de um grupo de empresas (as 100 maiores do mundo de acordo com a revista Fortune), utilizando o método da análise de conteúdo dos relatórios de sustentabilidade empresarial dessas empresas. Com base nas definições dos atributos de saliência propostos por Mitchell et al. (1997), esses autores criaram métricas qualitativas de análise de conteúdo para analisar os relatórios de sustentabilidade. Para tanto, usaram indicadores do campo do jornalismo para mensurar cada atributo. Para o atributo poder, Weber e Marley (2012) utilizaram três critérios: (a) qual stakeholder foi mencionado primeiro no relatório; (b) quais stakeholders foram discutidos de maneira explícita; (c) qual percentual de páginas no relatório foi dedicado para discutir a relação firma-stakeholder. Para o atributo legitimidade, os autores consideraram o mapa de stakeholders fornecido no relatório. Se um stakeholder estava presente nesse mapa, então consideravam para esse stakeholder o atributo legitimidade. Para o atributo urgência, caso um stakeholder nos relatórios analisados estivesse com uma prioridade com prazo explícito, ou o relatório expressasse que determinado stakeholder necessitava de atenção imediata, tal stakeholder era considerado como tendo o atributo de urgente. Para os três atributos e seus critérios de escolha, os autores atribuíram o valor "1" caso o stakeholder possuísse um dos atributos, e "0" caso não possuísse.

Bandiera et al. (2013) também empregaram técnicas de análise de conteúdo para analisar a saliência. Contudo, sua abordagem foi quantitativa uma vez que usaram a contagem de palavras para mensurar a saliência de um stakeholder. Como critério, os autores consideraram a distância de até 50 palavras entre o stakeholder e a palavra associada ao atributo. Assim, o número de incidências das palavras que representam um atributo próximo às palavras que representam um stakeholder foi a medida utilizada para mensurar se os atributos da saliência estavam associados a cada stakeholder (Bandiera et al.,2013). 


\section{METODOLOGIA DE ANÁLISE PROPOSTA}

Tendo em vista que o objetivo deste estudo é desenvolver um método de análise de conteúdo para analisar a saliência de stakeholders, a seção da metodologia é considerada, como a principal contribuição do estudo. A seção 4 corresponde aos resultados do emprego do método proposto, no sentido de testá-lo operacionalmente.

Segundo Bardin (2009), a análise de conteúdo pode ser de dois tipos distintos: análises quantitativas ou qualitativas. De forma geral, as análises quantitativas baseiam-se em técnicas de frequência das unidades de registro com a aplicação de técnicas estatísticas, ao passo que numa análise qualitativa as atenções estão focadas nas implicações decorrentes da mera presença ou ausência das unidades de registro em locais específicos da mensagem (Bardin, 2009). A escolha do tipo depende do conteúdo a ser analisado e do objetivo a ser alcançado. Nesta pesquisa, foi utilizada a análise de conteúdo quantitativa e o método proposto é composto por seis etapas, conforme apresentado na Figura 2 e explicado nas seções a seguir.

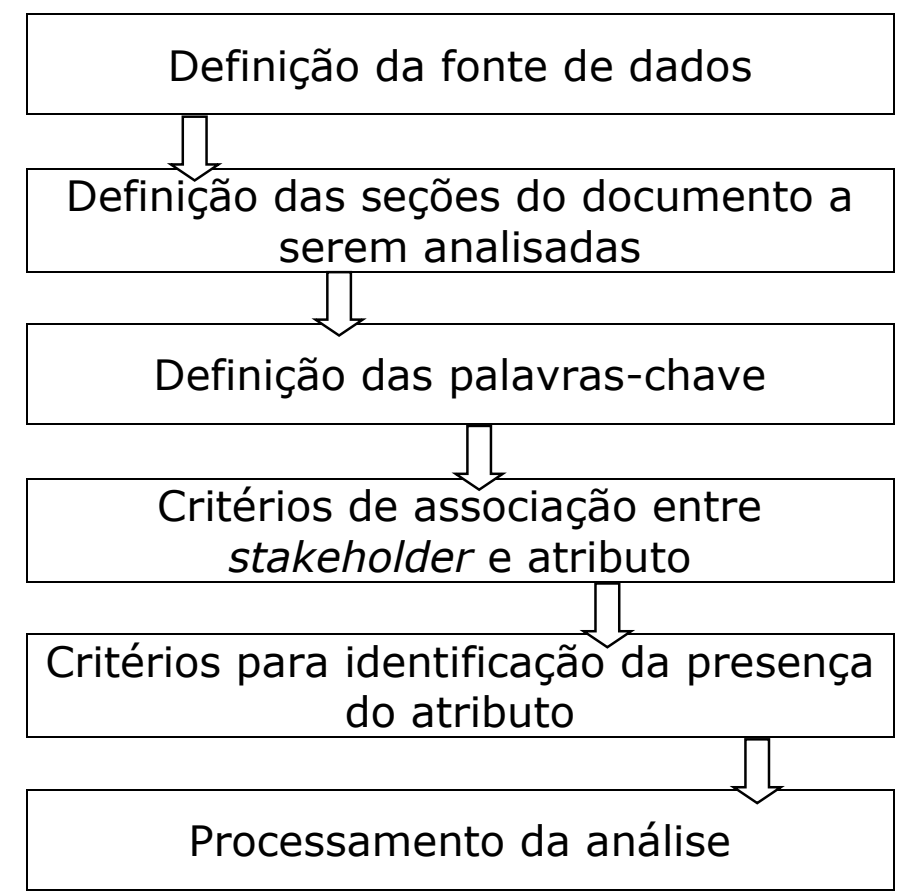

\section{Figura 2: Metodologia de análise}

Fonte: Autores 
Para analisar a consistência da metodologia proposta, os resultados obtidos na análise de conteúdo foram comparados, qualitativamente, às informações divulgadas nos sites das empresas analisadas.

\subsection{DEFINIÇÃO DA FONTE DE DADOS}

A primeira etapa da metodologia proposta é a escolha da fonte de dados. Para esta pesquisa, a coleta de dados foi realizada nos prospectos de distribuição pública inicial de ações. A opção pelos prospectos justifica-se pela riqueza de informações presente nesse relatório.

A decisão de abrir o capital é um dos eventos mais importantes no ciclo de vida de uma empresa e acontece no momento em que uma companhia vai ao mercado de capitais com o intuito de captar recursos (Oliveira, 2011).

Para executar o processo de abertura de capital, a empresa deve obedecer a alguns critérios definidos pela Comissão de Valores Mobiliários (CVM), órgão regulador do governo brasileiro do mercado acionário. Um desses critérios é a publicação do Prospecto de Distribuição Pública Inicial de Ações para o mercado, documento obrigatório, que contém informações relevantes para a tomada de decisão por parte dos investidores, conforme consta na instrução de no 400 da CVM (CVM, n.d.)

Art. 38 - Prospecto é o documento elaborado pelo ofertante em conjunto com a instituição líder da distribuição, obrigatório nas ofertas públicas de distribuição de que trata esta Instrução, e que contém informação completa, precisa, verdadeira, atual, clara, objetiva e necessária, em linguagem acessível, de modo que os investidores possam formar criteriosamente a sua decisão de investimento.

A CVM exige que no prospecto estejam as informações financeiras da companhia, detalhes sobre a distribuição de ações e todas as questões legais ligadas à oferta. Além dessas informações, a companhia também explicita como utilizará os recursos adquiridos com a abertura de capital, ou seja, sua estratégia futura. Todas as informações devem ser claras, objetivas e sem a omissão de fatos relevantes, sob pena de severas punições por parte da CVM caso qualquer infração ocorra.

Essas exigências legais de informações, além da própria intenção da empresa de abrir sua estratégia para o convencimento dos investidores, 
tornam o prospecto um documento exaustivo, que contém informações valiosas sobre a estratégia e as intenções da empresa.

Considerando que esta estratégia, em seus termos, indica 0 posicionamento da empresa para com seus stakeholders, o prospecto tornase uma fonte relevante de informação para a identificação da saliência dos stakeholders conforme percebida pelos gestores.

\subsection{DEFINIÇÃO DAS SEÇÕES DO DOCUMENTO A SEREM ANALISADAS}

Os relatórios empresariais, normalmente, são bastante extensos e apresentam as mais variadas informações, por isso, é importante definir as seções mais aderentes ao escopo de pesquisa.

O prospecto é um documento que pode chegar a mais de 500 páginas e possuir diversas seções, algumas focadas somente em questões legais, outras somente referentes aos bancos participantes da oferta, etc. Seções com informações jurídicas, de demonstração de resultados, com detalhes da oferta, entre outras, não são interessantes para uma análise de saliência, pois não fornecem informações sobre stakeholders. Além disso, se consideradas em uma análise de conteúdo, podem até enviesar a análise devido a seu conteúdo legal e centrado no acionista/investidor.

Portanto, para esta análise, serão consideradas apenas as seções do prospecto que contenham dados sobre a estratégia da companhia e relevantes para a identificação da saliência. Embora não haja uma padronização rigorosa do formato do prospecto, a CVM exige um rol de informações que devem estar presentes e destacadas nesses documentos.

Das informações exigidas pela CVM e presentes em todos os prospectos, é possível selecionar quais seções são interessantes para a análise de saliência. As seções que contêm informações sobre a estratégia e a companhia serão utilizadas para a análise, enquanto as seções sobre a oferta e informações financeiras não serão consideradas.

Com base na leitura dos prospectos, foram selecionadas as seções mais relevantes para a análise, que focam a atividade da companhia, seu mercado de atuação, indicações sobre estratégia futura e riscos atrelados à companhia e ao mercado. 
As seções escolhidas foram: (1) Sumário da Companhia - um resumo da companhia e de sua estratégia, localizado no início do prospecto; (2) Fatores de Risco - riscos levantados pela companhia em relação a seu negócio e à oferta de ações; (3) Análise setorial - dados sobre o mercado de atuação da companhia; e (4) Atividades - dados mais detalhados sobre a operação da companhia.

Exemplos de seções não consideradas: Sumário da Oferta, Informações Financeiras, Descrição do Capital Social, Dividendos e Política de Dividendos, etc.

Os prospectos considerados para análise foram de quatro empresas de setores diferentes da economia brasileira que realizaram a distribuição de ações entre 2006 e 2012. Foram selecionadas aleatoriamente distribuições com um intervalo de dois anos para evitar "modismos" de escrita da época dos prospectos. A escolha de empresas de setores diferentes foi também para evitar um viés de uso de determinado vocabulário típico de algum setor da economia.

Os prospectos selecionados foram: (1) Positivo Informática, ano de distribuição 2006, setor tecnologia da informação; (2) Le Lis Blanc, ano de distribuição 2008, setor varejo de roupas; (3) OSX, ano de distribuição 2010, setor petróleo e gás natural; e (4) Locamerica, ano de distribuição 2012, setor locação de automóveis. Todos os prospectos foram adquiridos no site da CVM (http://www.cvm.gov.br/).

A localização das seções consideradas para análise de cada prospecto foram as seguintes:

- Positivo: Sumário da Oferta - páginas 18 a 20; Fatores de Risco páginas 46 a 53; Análise Setorial - páginas 111 a 121; Atividades páginas 122 a 142 .

-OSX: Sumário da Oferta - páginas 14 a 22; Fatores de Risco páginas 70 a 84; Análise Setorial - páginas 120 a 145; Atividades páginas 146 a 176.

- Le Lis Blanc: Sumário da Oferta - páginas 17 a 20; Fatores de Risco - páginas 38 a 48; Análise Setorial - páginas 110 a 115; Atividades - páginas 116 a 142. 
- Locamerica: Sumário da Oferta - páginas 14 a 22; Fatores de Risco

- páginas 23 a 25; Análise Setorial e Atividades - não coberto.

\subsection{DEFINIÇÃO DAS PALAVRAS-CHAVE}

A terceira etapa da análise refere-se à identificação das palavras associadas a cada construto de análise.

O método quantitativo utilizado foi similar ao proposto por Bandiera et al. (2013) de identificação de palavras-chave. Porém, no presente estudo, a fonte utilizada para escolha das palavras-chave foi um dicionário analógico da língua portuguesa (Azevedo, 2010). A opção por essa fonte teve o objetivo de reduzir os critérios de subjetividade na escolha das palavras.

O dicionário analógico, ao invés de mostrar os significados para uma palavra conhecida, apresenta uma lista de palavras que contenham esse significado. É uma ferramenta muito utilizada por escritores e estudiosos da língua portuguesa, como o escritor e historiador Sérgio Buarque de Hollanda, como descrito no prólogo do dicionário analisado.

Esse dicionário classifica palavras da língua portuguesa e seus significados em áreas de uso (classes) e as ramifica em 24 subáreas (divisões). Cada divisão possui diversos grupos analógicos atrelados, que somam no total 1.000 grupos. O grupo analógico contém as palavras da língua portuguesa referentes ao conceito que representa.

Para este trabalho, foram escolhido grupos analógicos que correspondessem às definições, propostas por Mitchell et al. (1997), dos atributos poder, legitimidade e urgência. A escolha dos grupos analógicos por atributo é subjetiva, pois inúmeros grupos analógicos podem representar o mesmo conceito. Assim, dentre todo o universo de grupos analógicos, foram escolhidos aqueles que melhor representam as definições dos atributos.

No Quadro 1, apresenta-se um resumo das definições de cada atributo e suas bases, conforme proposto por Mitchell et al. (1997), assim como os grupos analógicos escolhidos para os três atributos de saliência. 


\begin{tabular}{|l|l|l|l|}
\hline Atributo & \multicolumn{1}{|c|}{ Definição } & \multicolumn{1}{c|}{$\begin{array}{c}\text { Grupos } \\
\text { Analógicos }\end{array}$} \\
\hline Poder & $\begin{array}{l}\text { Um relacionamento entre agentes sociais em que um } \\
\text { agente social A consegue que outro agente social B faça } \\
\text { algo que B não faria (Dahl, 1957; Pfeffer, 1981; Weber, } \\
1947, \text { citados por Mitchell et al., 1997, p. 865) }\end{array}$ & $\begin{array}{l}\text { Coercivo } \\
\text { Utilitário } \\
\text { Simbólico } \\
\text { (Etzioni, 1964) }\end{array}$ \\
\hline Legitimidade & $\begin{array}{l}\text { "Percepção generalizada ou uma presunção que as ações } \\
\text { de uma entidade são desejáveis, adequadas, ou } \\
\text { apropriadas dentro de um sistema social de normas, } \\
\text { valores, crenças e definições" (Suchman, 1995, p. 574, } \\
\text { citado por Mitchell et al., 1997, p. 866). }\end{array}$ & $\begin{array}{l}\text { Individual } \\
\text { Organizacional } \\
\text { Institucional } \\
\text { (Wood, 1991) }\end{array}$ \\
\hline Urgência & $\begin{array}{l}\text { "O grau em que a reivindicação do stakeholder demanda } \\
\text { atenção imediata" (Mitchell et al., 1997, p. 867). }\end{array}$ & $\begin{array}{l}\text { Sensibilidade a } \\
\text { Compelir } \\
\text { prazo } \\
\text { Criticidade }\end{array}$ \\
\hline
\end{tabular}

\section{Quadro 1: Grupos resumo das definições dos atributos}

As palavras-chave utilizadas na análise de conteúdo são aquelas que compõem os grupos analógicos. Por questões práticas, para que a lista de palavras não ficasse muito extensa, inviabilizando a pesquisa, foram escolhidos apenas quatro grupos analógicos por atributo.

Somente palavras consideradas como do "ambiente empresarial" foram selecionadas para análise. Por exemplo, palavras como "magnetizado" não foram consideradas, conforme apresentado no Quadro 2.

\begin{tabular}{|c|c|c|c|c|}
\hline Atributo & Classe & Divisão & Grupo Analógico & Palavras Escolhidas \\
\hline \multirow{4}{*}{\begin{tabular}{l}
$\frac{1}{2}$ \\
$\frac{D}{0}$ \\
\hdashline
\end{tabular}} & \multirow{2}{*}{$\begin{array}{l}\text { I. Relações } \\
\text { Abstratas }\end{array}$} & \multirow{2}{*}{ VIII. Causa } & Poder & $\begin{array}{l}\text { Poder, força, controle, competência, virtude, compelir, } \\
\text { poderoso, eficaz, eficiente, competente }\end{array}$ \\
\hline & & & Influência & $\begin{array}{l}\text { Pressão, domínio, influenciar, influente, popular, } \\
\text { conhecido }\end{array}$ \\
\hline & \multirow{2}{*}{ V. Vontade } & \multirow{2}{*}{$\begin{array}{l}\text { II. Com ref. } \\
\text { à sociedade }\end{array}$} & Autoridade & $\begin{array}{l}\text { Cargo, hierarquia, presidência, administração, } \\
\text { presidente, dominante, regente, dominador }\end{array}$ \\
\hline & & & Compelir & $\begin{array}{l}\text { Imposição, obrigação, vincular, forçar, obrigatório, } \\
\text { compulsório, compulsivo, formal, obrigatoriamente }\end{array}$ \\
\hline \multirow{4}{*}{ 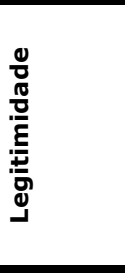 } & V. Vontade & $\begin{array}{l}\text { II. Com ref. } \\
\text { à sociedade }\end{array}$ & Contrato & $\begin{array}{l}\text { Contrato, transação, acordo, obrigação, compromisso, } \\
\text { condição, cláusula, negociação, contratar, contratual }\end{array}$ \\
\hline & \multirow{2}{*}{ VI. Afeições } & \multirow{2}{*}{ IV. Morais } & Direito & $\begin{array}{l}\text { Direito, autorização, constituição, reclamante, } \\
\text { reivindicador, recorrer, reivindicar, autorizado, justo }\end{array}$ \\
\hline & & & Legalidade & $\begin{array}{l}\text { Estatuto, estatutos, instituições, decreto, regulamento, } \\
\text { regulamentação, legal, devido, vigente }\end{array}$ \\
\hline & $\begin{array}{l}\text { IV. } \\
\text { Entendimento }\end{array}$ & $\begin{array}{l}\text { I. Formação } \\
\text { de Ideias }\end{array}$ & Crença & Segurança, confiança, seguro, capacitado \\
\hline \multirow{4}{*}{ 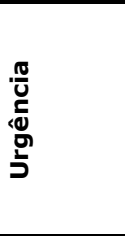 } & $\begin{array}{l}\text { I. Relações } \\
\text { Abstratas }\end{array}$ & VI. Tempo & Presteza & $\begin{array}{l}\text { Agilidade, pontualidade, velocidade, adiantamento, } \\
\text { urgência, antecipar, adiantar, pontual, antecipado }\end{array}$ \\
\hline & \multirow{3}{*}{ V. Vontade } & \multirow{3}{*}{ I. Individual } & Necessidade & $\begin{array}{l}\text { Essencial, principal, exigir, demandar, necessitar, útil, } \\
\text { urgente, insubstituível }\end{array}$ \\
\hline & & & Importância & $\begin{array}{l}\text { Relevância, fundamental, importante, relevante, vital, } \\
\text { fundamental, valioso, importância, proporção }\end{array}$ \\
\hline & & & Pressa & Urgência, tempo, apressado, imediatamente \\
\hline
\end{tabular}

Quadro 2: Palavras-chave por atributo 
Os stakeholders considerados foram os definidos como primários por Freeman (1984), sendo eles: acionistas, clientes, funcionários, fornecedores e governo.

Como as empresas podem denominar esses stakeholders com termos diferentes, foi feita uma pré-análise dos prospectos e identificados os termos utilizados por cada empresa para dirigir-se a esses grupos de stakeholders. Os termos considerados estão listados no Quadro 3.

\begin{tabular}{|l|l|l|l|l|}
\hline $\begin{array}{l}\text { Stakeholder / } \\
\text { Prospecto }\end{array}$ & Positivo & Le Lis Blanc & OSX & Locamerica \\
\hline Acionistas & Acionistas & Acionistas & Acionistas & Acionistas \\
\hline Funcionários & $\begin{array}{l}\text { Colaboradores, } \\
\text { funcionários }\end{array}$ & $\begin{array}{l}\text { Colaboradores, funcionários, } \\
\text { estilistas, vendedoras }\end{array}$ & $\begin{array}{l}\text { Colaboradores, } \\
\text { funcionários }\end{array}$ & $\begin{array}{l}\text { Colaboradores, } \\
\text { funcionários }\end{array}$ \\
\hline Clientes & $\begin{array}{l}\text { Clientes, } \\
\text { consumidores, } \\
\text { usuários, redes } \\
\text { varejistas }\end{array}$ & Clientes, consumidores & $\begin{array}{l}\text { Clientes, Petrobras, } \\
\text { OGX }\end{array}$ & Cliente, Clientes \\
\hline Fornecedores & Fornecedores & Fornecedores & $\begin{array}{l}\text { Fornecedor, } \\
\text { Fornecedores }\end{array}$ & $\begin{array}{l}\text { Fornecedores, } \\
\text { montadoras }\end{array}$ \\
\hline Governo & Governo & Governo & Governo & Governo \\
\hline
\end{tabular}

\section{Quadro 3: Palavras chave por stakeholder}

\subsection{CRITÉRIOS DE ASSOCIAÇÃO ENTRE STAKEHOLDER E ATRIBUTO}

Para uma palavra-chave ser vinculada a um stakeholder, ambos deveriam estar na mesma unidade de contexto analisada, conforme define Bardin (2009). A unidade de contexto considerada foi um parágrafo. Ou seja, para um stakeholder " $x$ " seria atribuído o atributo poder, por exemplo, caso as duas palavras estivessem no mesmo parágrafo.

A escolha do parágrafo como unidade de contexto foi subjetiva. $\mathrm{Na}$ alternativa a essa unidade de contexto, uma quantidade fixa de palavras antes e depois do stakeholder poderia ter sido usada, como na pesquisa de Bandiera et al. (2013). Contudo, agindo assim, corre-se o risco de identificar palavras que não necessariamente atribuem algo ao stakeholder. Por exemplo, em uma unidade de contexto de 50 palavras ao redor do stakeholder, caso esse termo estivesse no início ou final de um parágrafo, seriam atribuídas palavras do parágrafo anterior ou do subsequente a esse stakeholder. 


\subsection{CRITÉRIOS PARA IDENTIFICAÇÃO DA PRESENÇA DO ATRIBUTO}

Para analisar as palavras identificadas e atribuir a presença do atributo ao stakeholder, não era suficiente, apenas, a contagem de palavras. Por isso, a quantidade de parágrafos no cruzamento "stakeholder" $\mathrm{x}$ "atributo" foi comparada com o total de parágrafos identificados do stakeholder. Caso a leitura do atributo tenha ficado próxima ou maior a um percentual do total de parágrafos identificados, foi atribuído para esse stakeholder a presença do atributo de saliência. Para este trabalho, considerou-se como presente o atributo com $30 \%$ ou mais dos parágrafos indicados. A escolha desse valor foi arbitrária e parte de uma pré-análise dos dados obtidos.

\subsection{PROCESSAMENTO DA ANÁLISE}

Para a análise de conteúdo quantitativa foi utilizado o software de pesquisa QSR NVivo (http://www.qsrinternational.com/), que recebeu como input os seguintes dados: seções selecionadas dos prospectos (conforme descrito anteriormente), lista dos stakeholders primários e lista de palavraschave por atributo.

Como primeiro passo da análise, separaram-se os parágrafos que continham palavras relacionadas aos stakeholders primários. A identificação da unidade de contexto, o parágrafo, é feita automaticamente pelo software.

O segundo passo foi classificar por stakeholder, do total de parágrafos separados, quantos continham palavras-chave dos atributos de saliência.

\section{RESULTADOS DA APLICAÇÃO DO MODELO PROPOSTO}

Esta seção destina-se a apresentar os resultados do emprego do modelo metodológico proposto. Ressalta-se que esta aplicação tem como objetivo testar operacionalmente os critérios de análise descritos na metodologia para a análise da saliência. Por isso, a principal contribuição deste estudo está no desenvolvimento metodológico e não nos resultados em si. 
Nas tabelas e nos quadros a seguir, apresentam-se os resultados da análise quantitativa para cada empresa considerada na amostra. Nas Tabelas 1, 2, 3 e 4, apresenta-se o total de parágrafos em que um stakeholder estava associado a palavras do atributo e, nos Quadros 4, 5, 6 e 7, apresenta-se em que classificação do modelo de saliência o stakeholder se enquadrou, conforme apresentado na seção de referencial teórico. As linhas são os stakeholders; as colunas, os atributos de saliência; e os valores são a quantidade de parágrafos identificados para o stakeholder. Por exemplo, na Tabela 1, o valor de 5 no cruzamento da linha "Acionistas" com "Poder" significa que foram encontrados cinco parágrafos em que o stakeholder "Acionista" estava associado com palavras do atributo "Poder".

\subsection{RESULTADOS POSITIVO}

Na Tabela 1 e no Quadro 4, apresenta-se o resultado da análise da saliência dos stakeholders para a empresa Positivo.

Tabela 1: Contagem de palavras

\begin{tabular}{l|c|c|c|c}
\hline $\begin{array}{l}\text { Stakeholder/ } \\
\text { Atributo }\end{array}$ & Parágrafo & Poder & $\begin{array}{c}\text { Legitimi } \\
\text { dade }\end{array}$ & Urgência \\
\hline Acionista & 6 & 5 & 6 & 0 \\
\hline Cliente & 31 & 10 & 2 & 8 \\
\hline Fornecedor & 9 & 1 & 1 & 2 \\
\hline Funcionário & 15 & 2 & 1 & 2 \\
\hline Governo & 35 & 7 & 5 & 8 \\
\hline
\end{tabular}

\begin{tabular}{|l|c|c|c|l|}
\hline $\begin{array}{l}\text { Stakeholder/ } \\
\text { Atributo }\end{array}$ & Poder & $\begin{array}{l}\text { Legitimi } \\
\text { dade }\end{array}$ & Urgência & $\begin{array}{l}\text { Classe do } \\
\text { Stakeholder }\end{array}$ \\
\hline Acionista & SIM & SIM & NÃO & Dominante \\
\hline Cliente & SIM & NÃO & SIM & Perigoso \\
\hline Fornecedor & NÃO & NÃO & NÃO & Não Saliente \\
\hline Funcionário & NÃO & NÃO & NÃO & Não Saliente \\
\hline Governo & NÃO & NÃO & NÃO & Não Saliente \\
\hline
\end{tabular}

Quadro 4: Classificação do stakeholder 
$\mathrm{Na}$ análise do prospecto da empresa Positivo, os stakeholders que aparecem com mais frequência no relatório são Clientes e Governo. Mas, dos parágrafos em que foram identificados, proporcionalmente, em poucos foi encontrada a associação com algum atributo, o que resultou em uma baixa saliência para esses stakeholders. Acionistas, por outro lado, apresentou associação com palavras do atributo poder e legitimidade, resultando na classificação deste stakeholder como dominante.

A Positivo Informática é, hoje, a maior fabricante de computadores do Brasil e a número 1 em tecnologia educacional (Positivo, 2014). No site da empresa, é possível identificar informações sobre sua relação com clientes, funcionários, governo e acionistas, com destaque para este último grupo de stakeholders. A Positivo (2014) assim define sua missão: "Oferecer as melhores soluções de tecnologia, sempre com excelência em qualidade, custo e facilidade de uso, maximizando a criação de valor para os acionistas e as oportunidades de desenvolvimento para nossos colaboradores". Essa preocupação com acionistas, expressa em sua missão, é uma evidência da saliência deste stakeholder, o que está alinhado com os resultados desta pesquisa.

\subsection{RESULTADOS LE LIS BLANC}

Na Tabela 2 e no Quadro 5, apresenta-se o resultado da análise da saliência dos stakeholders para a empresa Le Lis Blanc.

Tabela 2: Contagem de palavras

\begin{tabular}{l|c|c|c|c}
\hline $\begin{array}{l}\text { Stakeholder } \\
\text { / Atributo }\end{array}$ & Parágrafo & Poder & $\begin{array}{l}\text { Legitimi } \\
\text { dade }\end{array}$ & Urgência \\
\hline Acionista & 12 & 7 & 5 & 1 \\
\hline Cliente & 22 & 14 & 10 & 6 \\
\hline Fornecedor & 46 & 4 & 20 & 2 \\
\hline Funcionário & 16 & 6 & 6 & 2 \\
\hline Governo & 8 & 3 & 0 & 2 \\
\hline
\end{tabular}




\begin{tabular}{|l|c|c|c|l|}
\hline $\begin{array}{l}\text { Stakeholder } \\
\text { /Atributo }\end{array}$ & Poder & $\begin{array}{l}\text { Legitimi } \\
\text { dade }\end{array}$ & Urgência & $\begin{array}{l}\text { Classe do } \\
\text { Stakeholder }\end{array}$ \\
\hline Acionista & SIM & SIM & NÃO & Dominante \\
\hline Cliente & SIM & SIM & SIM & Definitivo \\
\hline Fornecedor & NÃO & NÃO & NÃO & Não Saliente \\
\hline Funcionário & SIM & SIM & NÃO & Dominante \\
\hline Governo & SIM & NÃO & NÃO & Adormecido \\
\hline
\end{tabular}

Quadro 5: Classificação do stakeholder

No prospecto da empresa Le Lis Blanc, o stakeholder com maior quantidade de parágrafos foi Fornecedores, porém em poucos desses parágrafos foi possível associá-lo a um atributo, o que justifica sua classificação como Não Saliente. Clientes foi classificado como Definitivo, pois foi possível encontrar quantidade suficiente de cruzamentos dos três atributos nos parágrafos. Funcionários e Acionistas foram classificados como Dominantes, e Governo como Adormecido.

A marca Le Lis Blanc foi criada em 1988 por Waltraut Guida e Rahyja Afrange com o objetivo de desenvolver um negócio de moda feminina direcionada ao público de alto padrão aquisitivo (Le Lis Blanc, 2016). No site da empresa, observa-se grande preocupação com os clientes, como pode ser observado no trecho a seguir:

Le Lis Blanc é uma marca de alto padrão que traduz um estilo de vida moderno com um amplo portfólio de produtos, desde vestuário até decoração para casa, e conhecida por ser capaz de atender todas as necessidades da mulher, do dia a dia à roupa de festa. Em 2013, uma pesquisa divulgada pela revista Consumidor Moderno e feita junto a consumidores de alta renda colocou a Le Lis Blanc em $1^{\circ}$ lugar como marca de moda feminina mais amada do Brasil. A Le Lis Blanc também é comercializada para o público masculino por meio da linha Noir, Le Lis, além de possuir linhas para o público infantil, decoração para a casa, acessórios, cosméticos e maquiagem (Le Lis Blanc, 2016).

Os resultados desta pesquisa refletem essa posição da empresa, uma vez que o cliente foi o stakeholder com a maior saliência.

\subsection{RESULTADOS OSX}

Na Tabela 3 e no Quadro 6, apresenta-se o resultado da análise da saliência dos stakeholders para a empresa OSX. 
Tabela 3: Contagem de palavras

\begin{tabular}{l|c|c|c|c}
\hline $\begin{array}{l}\text { Stakeholder } \\
\text { /Atributo }\end{array}$ & Parágrafo & Poder & $\begin{array}{l}\text { Legitimi } \\
\text { dade }\end{array}$ & Urgência \\
\hline Acionista & 32 & 13 & 15 & 7 \\
\hline Cliente & 103 & 6 & 50 & 6 \\
\hline Fornecedor & 20 & 4 & 3 & 3 \\
\hline Funcionário & 9 & 6 & 2 & 4 \\
\hline Governo & 30 & 3 & 7 & 1 \\
\hline
\end{tabular}

\begin{tabular}{|l|c|c|c|l|}
\hline $\begin{array}{l}\text { Stakeholder } \\
\text { /Atributo }\end{array}$ & Poder & $\begin{array}{l}\text { Legitimi } \\
\text { dade }\end{array}$ & Urgência & $\begin{array}{l}\text { Classe do } \\
\text { Stakeholder }\end{array}$ \\
\hline Acionista & SIM & SIM & NÃO & Dominante \\
\hline Cliente & NÃO & SIM & NÃO & Arbitrário \\
\hline Fornecedor & NÃO & NÃO & NÃO & Não Saliente \\
\hline Funcionário & SIM & NÃO & SIM & Perigoso \\
\hline Governo & NÃO & SIM & NÃO & Arbitrário \\
\hline
\end{tabular}

\section{Quadro 6: Classificação do stakeholder}

Clientes foi o grupo de stakeholder com maior quantidade de parágrafos, 103 no total, contra 32 de Acionistas, a segunda maior quantidade. Porém o único atributo com identificações suficientes para esse stakeholder foi legitimidade, o que resultou em sua classificação como Arbitrário. Essa foi a mesma classificação de Governo. Acionistas foi classificado como Dominante; Funcionários, como Perigoso; e Fornecedores, como Não Saliente.

A OSX é a empresa do Grupo EBX que atua na indústria naval e offshore, oferecendo soluções de engenharia, construção, fretamento e serviços de operação e manutenção para empresas de exploração e produção de petróleo (OSX, 2014). Para esta empresa, os stakeholders mais salientes foram o acionista e o funcionário. Em visita ao site da empresa, observa-se uma preocupação mais acentuada com o acionista, uma vez que as opções de navegação apresentam mais informações quanto a relações com investidores e governança corporativa, o que pode caracterizar este stakeholder como dominante. Por outro lado, as informações sobre o 
stakeholder funcionário são pouco exploradas pelo site da empresa, o que pode indicar que os gestores percebem o stakeholder funcionário como Perigoso, segundo a classificação de Mitchell et al. (1997). Essas evidências corroboram os resultados desta pesquisa.

\subsection{RESULTADOS LOCAMERICA}

Na Tabela 4 e no Quadro 7, apresenta-se o resultado da análise da saliência dos stakeholders para a empresa Locamerica.

Tabela 4: Contagem de palavras

\begin{tabular}{l|c|c|c|c}
\hline $\begin{array}{l}\text { Stakeholder } \\
\text { / Atributo }\end{array}$ & Parágrafo & Poder & $\begin{array}{l}\text { Legitimi } \\
\text { dade }\end{array}$ & Urgência \\
\hline Acionista & 5 & 1 & 1 & 2 \\
\hline Cliente & 13 & 3 & 4 & 2 \\
\hline Fornecedor & 3 & 3 & 2 & 2 \\
\hline Funcionário & 2 & 0 & 0 & 0 \\
\hline Governo & 0 & 0 & 0 & 0 \\
\hline
\end{tabular}

\begin{tabular}{|l|c|c|c|l|}
\hline $\begin{array}{l}\text { Stakeholder } \\
\text { /Atributo }\end{array}$ & Poder & $\begin{array}{l}\text { Legitimi } \\
\text { dade }\end{array}$ & Urgência & $\begin{array}{l}\text { Classe do } \\
\text { Stakeholder }\end{array}$ \\
\hline Acionista & NÃO & NÃO & SIM & Reivindicador \\
\hline Cliente & NÃO & SIM & NÃO & Arbitrário \\
\hline Fornecedor & SIM & SIM & SIM & Definitivo \\
\hline Funcionário & NÃO & NÃO & NÃO & Não Saliente \\
\hline Governo & NÃO & NÃO & NÃO & Não Saliente \\
\hline
\end{tabular}

Quadro 7: Classificação do stakeholder

No prospecto da empresa Locamerica, não foram encontrados parágrafos com a palavra Governo, o que resultou em sua classificação como Não Saliente. Para Funcionários, foram identificados parágrafos, mas nenhum deles continha atributos de saliência, o que o tornou Não Saliente. O stakeholder Fornecedores, por outro lado, foi classificado como Definitivo, pois em quase todos os parágrafos identificados foi possível encontrar os atributos de saliência. Clientes foi classificado como Arbitrário; e Acionistas, como Reivindicador. 
A empresa Locamerica, especialista em locação de frotas para empresas, oferece customização, planejamento e gestão da frota para seus clientes. No site da empresa, há informações sobre sua capacidade financeira, nele a companhia indica que essa capacidade garante a seus clientes ótimas negociações e acordos comerciais com os fabricantes de veículos e peças. Além disso, a Locamerica diz comprar direto das montadoras e usar seu poder de negociação com fornecedores e seguradoras a favor do cliente (Locamerica, 2014). Essas informações contidas no site da empresa são evidências de que o cliente é visto como um stakeholder legítimo.

Embora a análise do prospecto tenha indicado que o stakeholder fornecedor apresente poder, legitimidade e urgência, observa-se que os trechos do site relacionados ao fornecedor apresentam uma relação de poder exercida pela empresa sobre o fornecedor. Esse resultado pode indicar uma limitação do método utilizado para o estudo do atributo poder, uma vez que análise de conteúdo não diferenciou se o poder é da empresa em relação ao stakeholder ou o contrário.

\section{CONSIDERAÇÕES FINAIS}

Nesta pesquisa, o objetivo foi desenvolver uma metodologia de análise de conteúdo para analisar a saliência de stakeholders, segundo o modelo de Mitchell et al. (1997). Para tanto, o processo metodológico foi composto por seis etapas, as quais incluíram: 1) definição da fonte de dados; 2) definição das seções do documento a serem analisadas; 3) definição das palavras-chave; 4) adoção de critérios de associação entre stakeholder e atributo; 5) adoção de critérios para identificação da presença do atributo; e, por fim, 6) processamento da análise. Após o processamento, os resultados foram comparados com informações divulgadas pelas empresas em seus websites.

Como resultado, observa-se que a metodologia é operacionalmente consistente, uma vez que foi possível identificar as classes de stakeholders propostas por Mitchell et al. (1997). As comparações dos resultados com as informações divulgadas nos sites indicaram que existe alinhamento. Assim, a 
pesquisa apresenta uma contribuição metodológica para a análise do modelo de saliência.

Além disso, o uso de documentos públicos, neste caso o prospecto, pode auxiliar os pesquisadores no acesso a maior quantidade de informações sobre o comportamento das empresas em relação aos stakeholders, o que talvez não fosse possível por meio de outra fonte de dados.

Neste estudo, alerta-se para alguns cuidados decorrentes de limitações e ensejam-se, em função disso, sugestões para estudos futuros.

A primeira limitação corresponde ao uso do prospecto como fonte de coleta de dados. Embora seja rico em informações sobre a empresa, esse documento não foi desenvolvido com o propósito de analisar a saliência dos stakeholders, o que não só pode causar algum viés na pesquisa, como também alguns prospectos podem não revelar todas as informações necessárias para uma análise plena. Esses pontos devem ser considerados na aplicação de alguma pesquisa futura. Essa limitação é, também, apontada por Bandiera et al. (2013).

A segunda limitação refere-se ao tamanho da amostra, bastante reduzido, quatro casos, assim, uma replicação em amostra maior de casos poderá ampliar o conhecimento do método.

Um terceiro ponto é quanto à análise do atributo poder, que não pôde ser avaliado com precisão, ou seja, se é exercido pela empresa ou pelo stakeholder. Este ponto pode ser objeto de novos estudos que refinem o método e superem essa dificuldade.

\section{REFERÊNCIAS}

Agle, b. R., Mitchell, r. K., \& Sonnenfeld, J. A. (1999). Who matters to Ceos? An investigation of stakeholder attributes and salience, corpate performance, and Ceo values. Academy of Management Journal, 42(5), 507-525.

Azevedo, F. F. S. (2010). Dicionário analógico da língua portuguesa (2a ed.). Rio de Janeiro: Lexikon.

Bandiera, E. G., Boaventura, J. M. G., Mascena, K. M. C. de, \& Fischmann, A. A. (2013). Saliência de stakeholders e sua relação com a indústria: um estudo em empresas brasileiras de capital aberto. Revista de Finanças Aplicadas, 1, 1-23. 
Bardin, L. (2009). Análise de conteúdo. Lisboa: Edições 70.

Clarkson, M. E. (1995). A stakeholder framework for analyzing and evaluating corporate social performance. Academy of Management Review, 20(1), 92-117.

Comissão de Valores Mobiliários - CVM. (n.d.). Instrução CVM no 400. Recuperado em 12 de dezembro, 2013, de http://www.cvm.gov.br/asp/cvmwww/atos/exiato.asp?File=/inst/inst400. htm

Dahl, R. A. (1957, July). The concept of power. Behavioral Science, 2(3), 201-215.

Donaldson, T., Preston, L. E. (1995). The stakeholder theory of the corporation: concepts, evidence, and implications. Academy of Management Review, 20(1), 65-91.

Etzioni, A. (1964). Modern organizations. New jersey: Prentice-Hall.

Freeman, R. E. (1984). Strategic management: a stakeholder approach. Boston: Pitman.

Le Lis Blanc. (2016). Recuperado em 1 de abril, 2016, de http://www.restoque.com.br/restoque/web/conteudo_pt.asp?idioma=0\&t ipo $=41556 \&$ conta $=28 \&$ id $=154055$

Locamerica. (2014). Recuperado em 14 de abril, 2014, de http://www.locamerica.com.br/Locamerica/missao_e_valores.aspx

Mitchell, R. K., Agle, B. R., \& Wood, D. J. (1997). Toward a theory of stakeholder identification and salience: defining the principle of who and what really counts. Academy of Management Review, 22(4), 853-886.

Neville, B. A., Bell, S. J., \& Whitwell, G. J. (2011). Stakeholder salience revisited: refining, redefining, and refueling an underdeveloped conceptual tool. Journal of Business Ethics, 102(3), 357-378.

Oliveira, B. C. de. (2011). Fatores determinantes para abertura de capital de empresas brasileiras. Dissertação de Mestrado, Faculdade de Economia, Administração e Contabilidade da Universidade de São Paulo, SP, Brasil.

OSX. (2014). Recuperado em 14 de abril, 2014, de http://www.osx.com.br

Parent, M. M., Deephouse, D. L. (2007). A case study of stakeholder identification and prioritization by managers. Journal of Business Ethics, 75(1), 1-23.

Phillips, R. A., \& Reichart, J. (2000). The environment as a stakeholder? A fairness-based approach. Journal of Business Ethics, 23(2), 185-197. 
Positivo (2014). Recuperado em 14 de abril, 2014, de http://ri.positivoinformatica.com.br/positivo/web/conteudo_pt.asp?idiom $\mathrm{a}=0$ \&conta $=28 \&$ tipo $=29309$

Suchman, M. C. (1995). Managing legitimacy: strategic and institutional approaches. Academy of Management Review, 20(3), 571-610.

Xavier, D. L., Vieira, S. F. A., \& Costa, B. K. (2011). Análise do stakeholder pelo método de saliência: a caso de um banco de crédito consignado. Revista de Administração FACES Journal, 10(2), 165-185.

Weber, M. (1947). The theory of social and economic organization. New York: Oxford University Press.

Weber, J., \& Marley, K. A. (2012). In search of stakeholder salience: exploring corporate social and sustainability reports. Business \& Society, 51(4), 626649.

Wood, D. J. (1991). Corporate social performance revisited. Academy of Management Review, 16(4), 691-718. 